\title{
Perspective
}

Actualité en histoire de l'art

\section{L'histoire de la photographie sociale et documentaire dans l'entre-deux-guerres. Paris dans le contexte transnational}

The History of Social and Documentary Photography in the Inter-war Period.

Paris in the Transnational Context

Die Geschichte der Sozial- und Dokumentarfotografie zwischen den Kriegen.

Paris im transnationalen Kontext

La storia della fotografia sociale e documentaristica tra le due Guerre. Parigi nel contesto transnazionale

La historia de la fotografía social y documental durante el periodo de entreguerras. Paris en el contexto transnacional

\section{Christian Joschke}

\section{OpenEdition}

Journals

Édition électronique

URL : https://journals.openedition.org/perspective/7197

DOI : $10.4000 /$ perspective. 7197

ISSN : 2269-7721

Éditeur

Institut national d'histoire de l'art

Édition imprimée

Date de publication : 30 juin 2017

Pagination : 113-128

ISBN : 9782917902387

ISSN : 1777-7852

Référence électronique

Christian Joschke, «L'histoire de la photographie sociale et documentaire dans l'entre-deux-guerres. Paris dans le contexte transnational », Perspective [En ligne], 1 | 2017, mis en ligne le 31 décembre 2017, consulté le 16 janvier 2023. URL : http://journals.openedition.org/perspective/7197 ; DOI : https://doi.org/10.4000/perspective.7197 
Christian Joschke

\section{L'histoire de la photographie sociale et documentaire dans l'entre-deux-guerres. Paris dans le contexte transnational}

Si le soupçon d'un asservissement de l'art à la politique a souvent plané sur la figure de l' " artiste engagé ", il en est autrement du photographe documentaire. Nouveau héros des temps modernes, il a su tirer de ses luttes politiques et sociales un ethos, une pratique et une morale artistiques nouvelles qui articulent indissociablement la question de la forme et celle du contenu politique. Par le traitement visuel des sujets sociaux s'est inventé un langage : le pavé parisien, le portrait d'anonymes, le corps du travailleur, les masses agissantes, le peuple souffrant sous les bombardements d'Espagne, l'enfance ou le rêve d'un bonheur projeté sur une nouvelle génération, tous ces thèmes ont fourni la matière d'une esthétique nouvelle de l'image prise sur le vif, mouvante, fragmentaire, déclinant en d'infinies variations les effets du dispositif photographique. Sur ce registre s'est déployé et aiguisé le regard des photographes que l'on a associés aux luttes pour le progrès social.

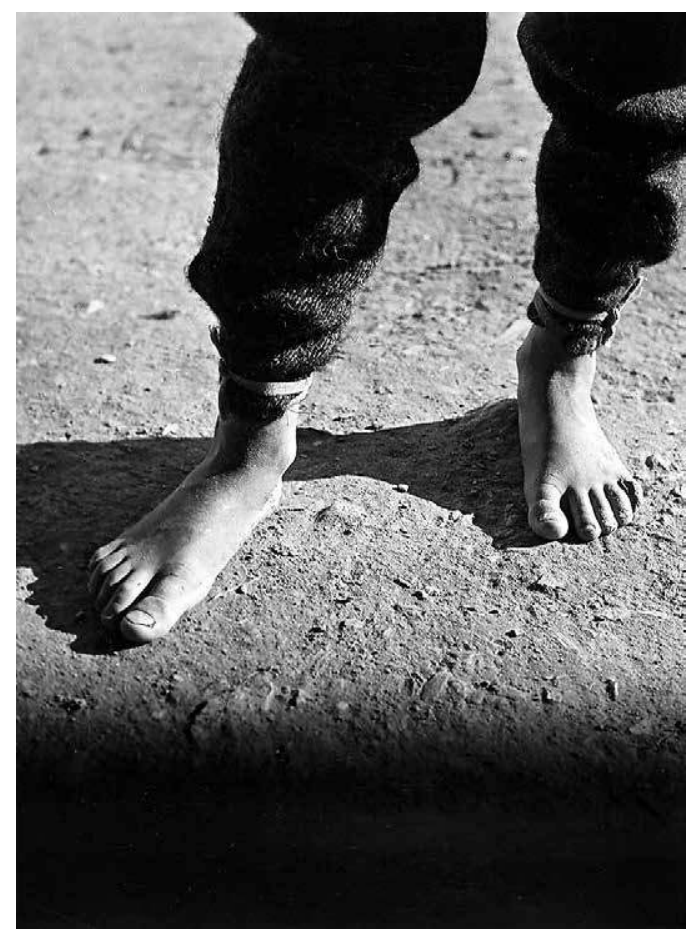

1. Aurel Bauh, Pieds, vers 1930, épreuve gélatinoargentique, Paris, Collection Centre Pompidou, Musée national d'art moderne - Centre de création industrielle. L'historiographie a célébré ces figures de l'engagement. De Henri Cartier-Bresson à David Seymour, de Robert Capa à Willy Ronis, on admire l'audace du regard, la fibre humaniste, l'amour du peuple.

Aussi n'a-t-on pas hésité, sur la base de ces biographies exemplaires, à situer l'origine de ce regard dans l'expérience du Front populaire (DENOYELLE, Cuel, VIBERT-Guigne, 2006). L'essor d'une culture pour tous, la révolution des congés payés, les grandes manifestations :

Co-directeur de Transbordeur, nouvelle revue d'histoire de la photographie, Christian Joschke enseigne I'histoire de I'art contemporain et de la photographie à l'université Paris Nanterre. Il conduit actuellement le projet «Photographie sociale et documentaire des années 1930 » (université Paris Nanterre/Centre Pompidou/Labex Arts H2H). 
2. "À Berlin», première de couverture de $V U, 4^{\mathrm{e}}$ année, $\mathrm{n}^{\circ} 179,19$ août 1931.

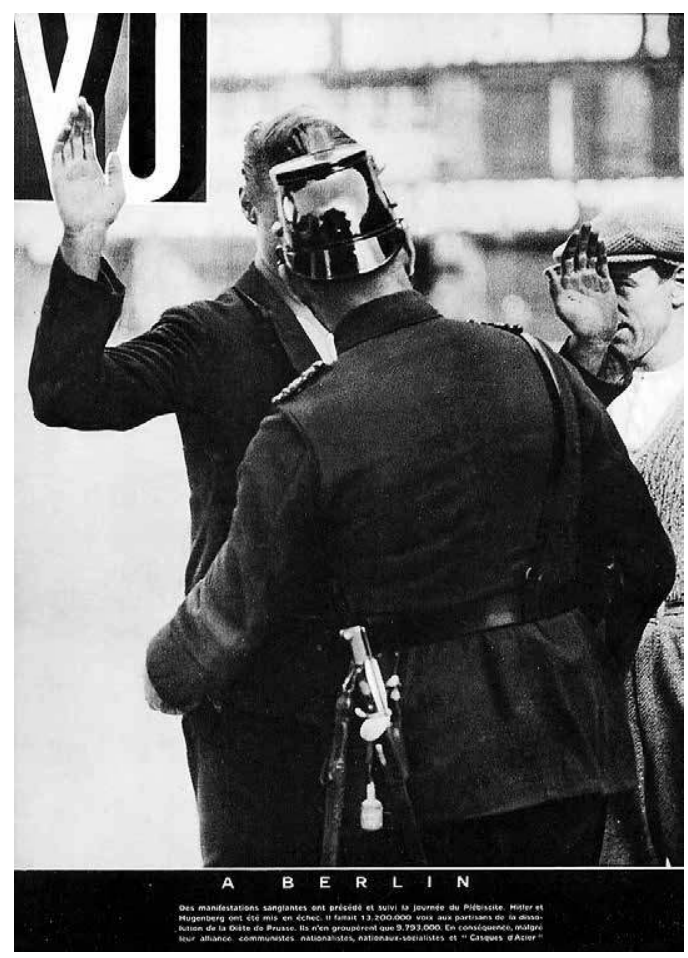

autant de sujets célébrés par ces " génies » de la photographie. Avec la guerre d'Espagne, le regard se précise. Au peuple jovial des loisirs parisiens s'oppose désormais le peuple souffrant, victime du franquisme et de l'alliance fasciste. C'est là que se forge une culture du reportage au plus près de l'événement, symbolisé par le maniement de l'appareil petit format à visée directe. C'est aussi là que s'invente une nouvelle gestion des images : la coopérative de photographes. Sur les vestiges d'Alliance photo, créé en 1934 autour de Maria Eisner et René Zuber, naîtra en 1947 l'agence Magnum, reprenant le même principe d'une valorisation des auteurs dans l'exploitation des clichés (DenOyelle, 1997 ; Molderings, 1980). Moment doublement fondateur, l'année 1936 est donc restée dans nos esprits comme l'origine tant de la "photographie humaniste " que du reportage de guerre.

Or à trop célébrer le " génie " des photographes, on oublie sans doute le rôle catalyseur des structures collectives et des expériences militantes qui ont préparé cet avènement d'une culture visuelle populaire. C'est qu'avant d'être humaniste, la photographie a été sociale. Il ne faut en effet pas confondre l'une et l'autre. La photographie sociale plonge ses racines dans l'action politique et les structures de la gauche radicale des années 1920 et 1930. La photographie humaniste se développa dans le contexte de l'après-guerre, portée sur le plan international par l'exposition " Family of Man " (LUGON, 2009) et, en France, par les photographes comme Robert Doisneau et Willy Ronis (La photographie humaniste..., 2006). Et si l'historiographie a souvent considéré l'entre-deux-guerres comme une prémisse de l'humanisme libéral d'après-guerre, c'est qu'elle se fonde avant tout sur l'expérience du Front populaire. Les travaux plus récents tendent au contraire à souligner la spécificité de la première période, antérieure à 1936. Elle fut pour bon nombre de photographes, graphistes (même si le terme n'existe pas encore), hommes de presse, artistes, des années de formation, comme un atelier du regard social. On peut aisément prendre comme terminus post quem la date de 1928, moment de fondation de deux magazines illustrés essentiels pour la culture visuelle politique de la décennie suivante : $V U$ (fig. 2) et Regards. C'est dans ces rédactions qu'ont été expérimentées des formules iconographiques - comme la plongée et la contre-plongée, la métonymie du corps, la vue au ras du sol - importées des recettes graphiques venues d'ailleurs ; c'est là qu'a été forgée une conscience politique du regard. On ne voit pas le monde de la même façon dans l'Illustration d'Emmanuel Sougez et dans $V U$ de Carlo Rim et Maximilien Vox, où l'on célèbre avec un attachement mâtiné d'ironie bienveillante les "philosophes " du pavé parisien, où l'on enquête sur le logement, où l'on rend compte de la soupe populaire, des abattoirs de la Villette ou de l'Institut des sourds et muets d'Asnières (LAVIE, 2013 ; Eli Lotar, 2017). Aux structures de la presse s'ajoutèrent rapidement les structures culturelles et politiques de la gauche radicale avec la création des Amateurs photographes ouvriers (1930) et, un peu plus tard, l'Association des écrivains et artistes révolutionnaires - AER en 1931, puis AEAR en 1932 - qui, engagée à absorber une partie du groupe surréaliste, 
n'en combattait pas moins les principes au nom du réalisme socialiste (ALLAIN, 2015). Entre 1928 et 1936 s'est donc créé un vivier de photographes aguerris à la question sociale, côtoyant les militants, attachés à traduire en image les combats politiques.

Si l'historiographie a minimisé la part de la photographie sociale dans la fin des années 1920, c'est qu'elle a associé cette période en priorité à deux évolutions de la culture visuelle parisienne qui ont été peu mises en rapport avec le contexte des luttes politiques : l'émergence d'une modernité photographique et les engagements pour une reconnaissance culturelle du métier de photographe-artiste. Il suffit, pour s'en faire une idée, de rappeler les textes de critiques d'art évoqués par Quentin Bajac dans Voici Paris, qui insistent, à l'instar de Waldemar George, sur la seconde naissance de la photographie : "Après avoir été inventée il y a de cela un siècle, la photographie n'aurait été découverte dans toutes ses possibilités artistiques qu'au tournant des années 1920 "(BAJAC, 2012). Michel Frizot a étudié en profondeur les ressorts du photomontage et de la nouvelle vision, insistant sur son lien avec la presse illustrée (FRIZOT, 2014 et 2015). Il faut y ajouter les spécificités d'un surréalisme friand d'expérimentations formelles, de déformations, de solarisations, de brûlages et autres incongruités photographiques (POIVERT, 2006 ; Voici Paris..., 2012-2013 ; MOLDERINGS, 2009). Les années 1928-1936 sont celles d'une photographie moderne et formaliste marquée par l'abstraction, auxquelles succèderait l'invention du regard humaniste dominé par le portrait et la figure humaine.

Il convient néanmoins aujourd'hui d'étudier la spécificité de cette même période sous l'angle documentaire si l'on ne veut pas donner, comme le suggère la séparation trop nette de deux champs de l'historiographie, une image trop contrastée de l'ensemble où l'avant 1936 serait associé à l'invention de la modernité et l'après 1936 à la découverte de l' " humanisme ". Ce qui se joue dans cette dynamique de préfiguration ne se laisse pas mesurer à la seule échelle des modernes et des antimodernes. C'est l'idée tout sociale qu'un art moyen invente ses manières de capturer le monde, plus proche des chantiers et des chaînes de montage ; qu'un renversement symbolique s'opère dès lors que les outils de production visuelle peuvent aussi être transmis directement à la classe ouvrière. La réévaluation de cette période a déjà débuté, en France comme à l'étranger. Elle consiste en un triple déplacement : vers l'histoire de la presse et du cinéma ; vers l'histoire transnationale ; vers les autres producteurs, les photographes amateurs. Ce déplacement des enjeux situe désormais la question formelle dans un contexte plus large, où les données culturelles, sociales, techniques viennent fournir au langage des formes un statut nouveau, comme l'expression collective de mutations sociales et politiques.

\section{La photographie sociale dans le contexte de la presse illustrée}

Pour comprendre l'émergence de ce nouveau regard social, différent à bien des égards de la philanthropie d'avant-guerre, il faut se rendre compte de l'interpénétration entre les modernités photographiques et les sujets sociaux. L'attention des historiens s'est longtemps focalisée sur les productions surréalistes de André Kertész, jacques-André Boiffard, Eli Lotar, Brassaï et sur la nouvelle vision de Germaine Krull dans sa série Métal, en négligeant toutefois leur importante contribution au reportage social, aujourd'hui étudiée : la série de Krull sur la soupe populaire, celle de Marianne Breslauer sur les clochards de Paris, la contribution de Kertész à l'AEAR (DENOYELLE, CUEL, VIBERT-GUIGNE, 2006). Le renouvellement des questionnements autour de l'œuvre de Henri Cartier-Bresson s'appuie sur un aspect longtemps mis en retrait de sa biographie, à savoir sa fréquentation des milieux communistes, son amitié avec Henri Tracol, 
3. Henri

Cartier-Bresson,

Le Couronnement

de George VI,

12 mai 1937 ,

épreuve gélatino-

argentique,

Paris, Collection

Centre

Pompidou,

Musée national

d'art moderne -

Centre de

création

industrielle.

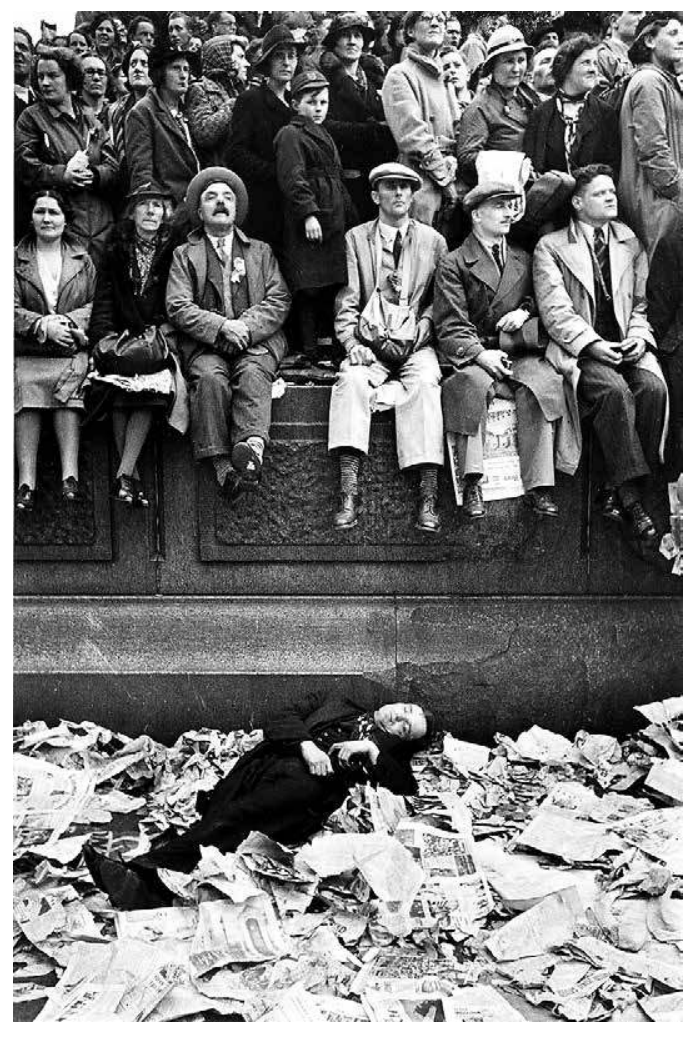

figure oubliée de la photographie militante, et son travail pour les plateaux de tournage, en particulier auprès de Jean Renoir (FRIZOT, 2010 ; Henri Cartier-Bresson, 2013). C'est là que s'aiguise une conscience du peuple subtilement mêlée aux habitudes des surréalistes, que l'on saisit avec bonheur dans les nus, les drapés, ou les regards. La série sur le couronnement du roi George VI à Londres, où il photographie les regards de la foule devant le cortège, préférant l'hommage au peuple à la célébration des puissants, prend sur ce fond un nouvel éclairage (fig. 3). Il n'y a pas de contradiction entre l'amour des sujets insolites du Paris des années 1920 et ce regard engagé, tantôt bienveillant à l'égard des ouvriers, tantôt indigné par la misère. La rue, les vitrines des magasins, les détritus retournés par les clochards, le travail des terrassiers et des paveurs, ces sujets sont souvent l'occasion d'un mariage entre les thèmes surréalistes de l'inquiétante étrangeté - cette vision du même devenu autre - et la mise au jour des injustices du capitalisme (AMAO, 2014, Eli Lotar, 2017). Les nuits parisiennes de Brassaï, le langage des graffitis, expression populaire et acte d'insoumission par excellence (Brassaï, graffiti..., 2016), les trottoirs des Halles, la sieste sur les quais, les cafés de la zone. Le document du monde social prend des allures d'étrangeté quand il apparaît dans Documents, la revue de Bataille, ou dans La Révolution surréaliste de Breton, tandis qu'il parle un langage clair et pédagogique quand il est publié dans VU, Regards ou Ce Soir (AMAO, 2014, Eli Lotar, 2017).

Cette interpénétration de la modernité et du reportage social apparaît clairement quand on s'intéresse à la presse illustrée, dont Michel Frizot a été le premier à renouveler les études (FrIZOT, VEIGY, 2009 ; FRIZOT, 2010 ; 2014 et 2015). Ce n'est pas minimiser le talent des photographes que de dire que c'est elle qui, à travers ses différents métiers, a formé le cadre opératoire de leur pratique, que la vie d'un cliché doit autant à l'opérateur qu'aux responsables éditoriaux, artistiques, aux typographes et imprimeurs, et que l'un des débouchés essentiels des photographes dans l'entre-deux-guerres est la presse et le livre. Longtemps laissée aux mains des historiens des médias (D'ALMEIDA, DELPORTE, 2003) qui l'ont enrichie d'un savoir précieux sur les structures et les discours des médias contemporains, l'histoire de la presse émerge aujourd'hui comme une branche importante de l'histoire de la photographie (FRIZOT, 2010 ; GERVAIS, 2008 et GERVAIS, MOREL, 2015). Elle nous apprend par exemple que la charge politique d'un organe de presse ne se mesure pas seulement au contenu des messages "politiques ", à l'engagement de tel ou tel photographe mais aux structures du photojournalisme, et au statut qu'on donnait aux images dans les magazines illustrés. Le cas du magazine communiste allemand Arbeiter Illustrierte Zeitung est particulièrement éclairant. Au "mensonge " des agences bourgeoises, il lui fallait opposer un modèle fondé sur le « regard de classe » (fig. 4). Comme l'a montré Andrés Mario Zervigón, le rejet de ce qui était considéré comme une propagande capitaliste conduisait, dans l'AIZ, à une déconstruction critique 

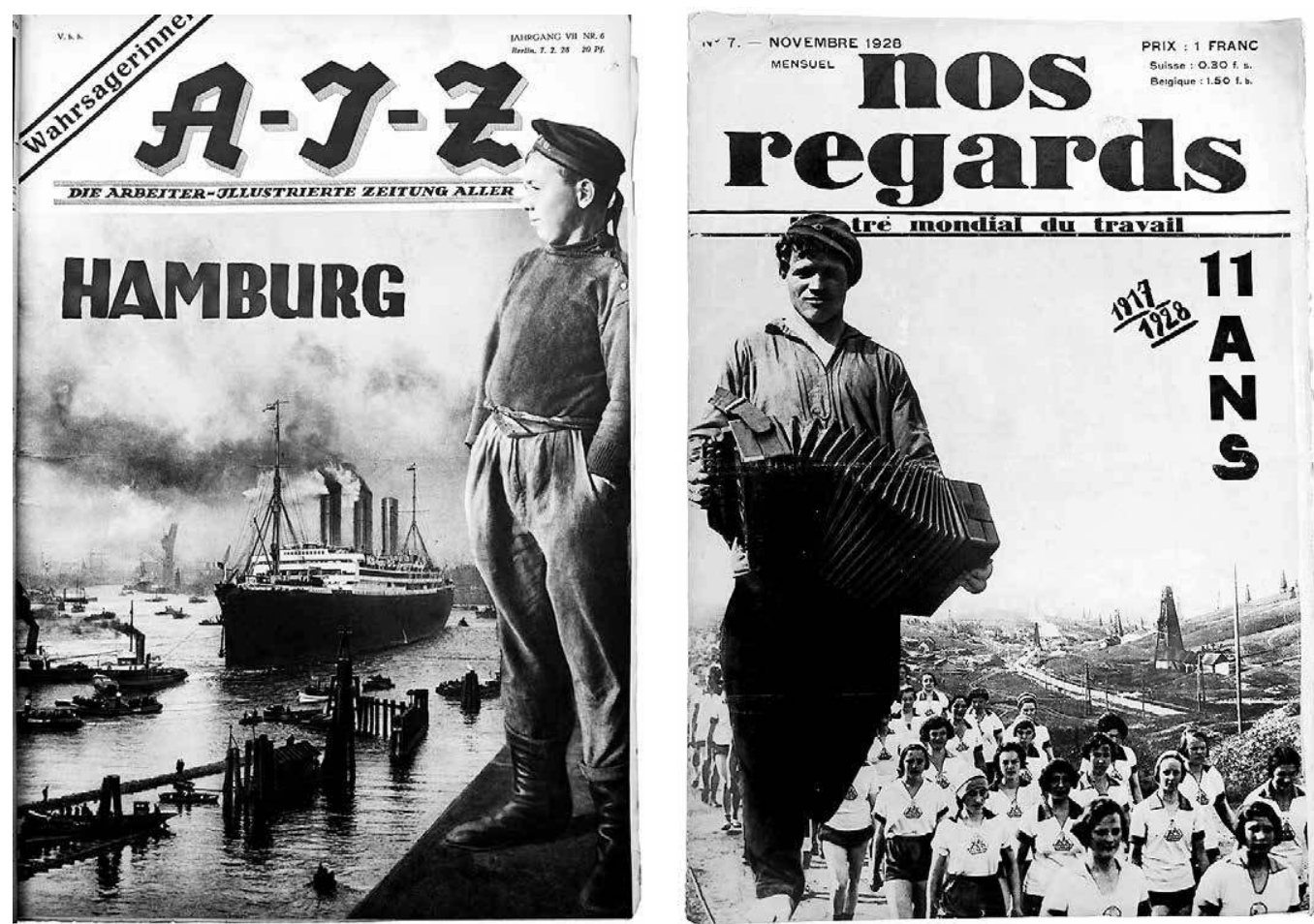

4. Première de couverture de l'Arbeiter Illustrierte Zeitung de février 1928, $\mathrm{n}^{\circ} 6$.

5. Première de couverture de Nos Regards. Illustré mondial du travail, $\mathrm{n}^{\circ} 7$, novembre 1928.
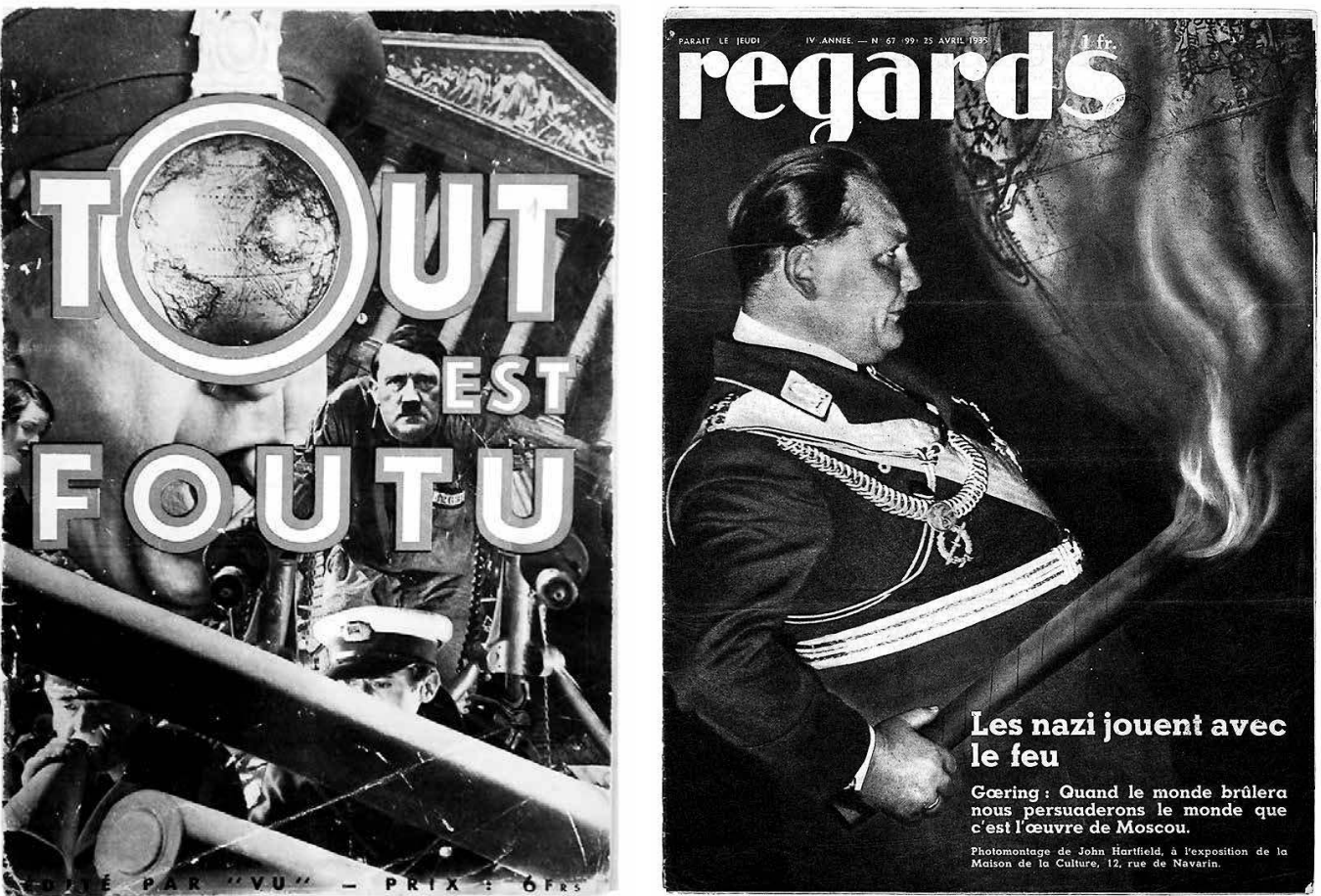

6. Carlo Rim et Maximilien Vox Tout est Foutu, supplément à VU, 23 avril 1932, première de couverture.

7. John Heartfield, «Quand le monde brûlera, nous persuaderons le monde que c'est l'œuvre de Moscou ",

première de couverture de Regards, n ${ }^{\circ} 66$, 25 avril 1935. 
du langage visuel de la " presse bourgeoise " (notamment les Münchner Illustrierte Zeitung et Berliner Illustrierte Zeitung) faite de montages visuels et de légendes en contre-point visant à discréditer les figures du pouvoir politique et économique de la République de Weimar (ZERVIGÓN, 2010). La critique des documents visuels du capitalisme, critique des images par les images, tel était l'objectif assumé des rédacteurs du célèbre magazine communiste, en réponse à une méfiance exprimée par les cadres du Parti communiste allemand à l'égard des images, ce que Zervigón appelle une "iconophobie communiste ".

Le modèle critique allemand fut d'ailleurs adapté en diverses occasions, d'abord pour Regards (fig. 5 ; MOREL, 2001), dont on oublie souvent qu'il n'a pas été créé en 1932 par le Parti communiste français, mais en 1928 par le Secours ouvrier international et les éditeurs de l'AIZ. On retrouve ce style consistant à faire parler la dialectique des images notamment chez Carlo Rim et Maximilien Vox, dans une brochure pleine d'ironie publiée en 1930 et intitulée Tout est foutu (fig. 6 ; FRIZOT, VEIGY, 2009). C'est aussi dans ces organes que s'épanouit le photomontage politique. Soulignons le regain d'intérêt que suscite aujourd'hui chez les historiens de l'art la figure de John Heartfield, dont l'influence mondiale se fera sentir dès le milieu des années 1930. Zervigón a enquêté sur ses premières années d'activité, entre 1913 et 1929, et situe le concept d' "image d'agitation » dans une filiation multiple qui va du théâtre et de la performance à l'affiche politique. Il rend ainsi caduque l'idée selon laquelle l'« invention " du photomontage par l'avant-garde dadaïste aurait été " adaptée " à des objectifs politiques (ZERVIGÓN, 2012). Au contraire, le personnage de John Heartfield semble avoir été animé, dès ses jeunes années, par la volonté de produire des œuvres frappantes, à la mesure de son indignation sociale (fig. 7). Sabine Kriebel, quant à elle, place le photomonteur dans le contexte des années 1930, dans son rapport ambigu au parti communiste allemand et à l'URSS : la doctrine mise en place par Jdanov condamnait fermement la satire comme outil politique, et s'opposait de ce fait à la politique culturelle menée dans la décennie précédente par Lounatcharski (KRIEBEL, 2014). John Heartfield est néanmoins célébré à Paris en 1935 , par Aragon notamment, qui perçoit en lui un art qui s'adresse aux masses, ayant fait de la presse illustrée son principal support. L'image-critique, l'ironie du montage, l'acidité du verbe et de la photographie, voilà sans doute ce qui caractérise la spécificité d'un regard politique dans la très influente AIZ.

L'autre réponse à l'hégémonie des " agences bourgeoises " fut de chercher à produire une illustration authentiquement ouvrière en se passant tout simplement du service des grandes agences. Cette exigence pouvait se traduire par le recours à des amateurs, nous y reviendrons. L'emploi de photographes indépendants dans $V U$ et plus tard dans Ce Soir - autre stratégie pour gagner une indépendance par rapport à la livraison quotidienne des agences - n'est certes pas un procédé réservé aux journaux de gauche. Mais il permet néanmoins d'orienter politiquement les sujets, de produire des reportages en dehors du flux quotidien des images ${ }^{1}$. Ce n'est que dans un second temps que Regards emboîta le pas à la stratégie de $V U$, employant lui aussi des photographes indépendants et publiant, cette fois sur le modèle soviétique, des " essais photographiques", véritables numéros thématiques du journal (WOLF, 2011).

Michel Frizot a déjà mis en évidence la proximité de la presse illustrée avec le cinéma (FRIZOT, 2010). Les effets de dynamisme visuel de la mise en page, les jeux de champ/contrechamp, de plongée/contre-plongée qui font apparaître chaque image comme un appel de la suivante sont autant d'éléments issus du " modèle cinéma ". Il n'est pas inutile de rappeler le service rendu au cinéma par les photographes : Germaine Krull, Eli Lotar et Joris Ivens ont étroitement collaboré, comme le rappelle Damarice Amao dans l'exposition du Jeu de Paume (Eli Lotar, 2017). Avec l'essor de la fonction d'opérateur (VezYroglou, 2012), la miniaturisation des caméras (notamment avec la Kinamo), un regard-cinéma s'impose progressivement dans les pratiques photographiques. S'ajoute, dans le cas de Lotar ou de Krull, l'inscription 

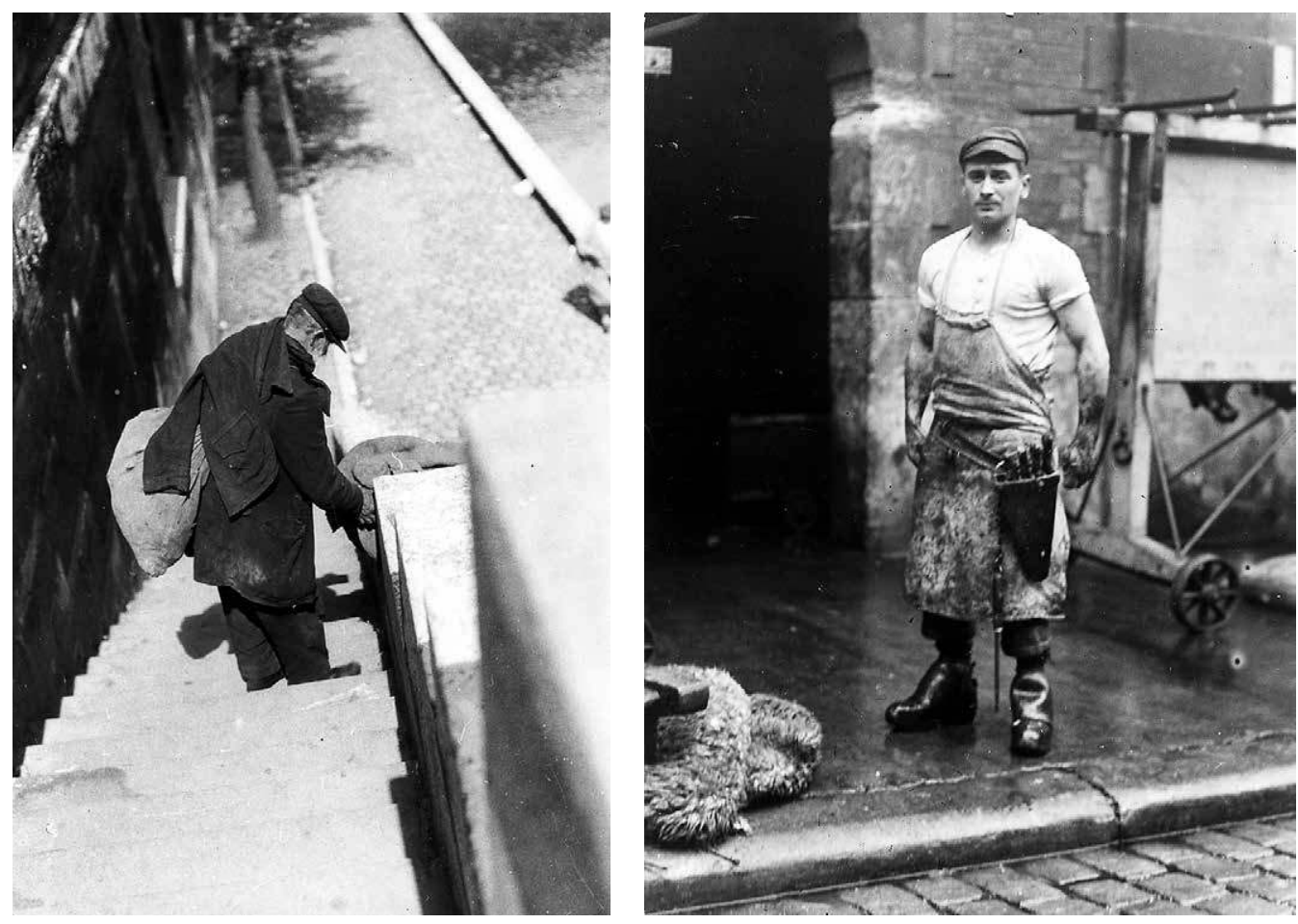

8. Germaine Krull, Clochard, 1928, épreuve gelatinoargentique, Essen, Museum Folkwang.

9. Eli Lotar, Aux abattoirs de la Villette,

1929, négatif sur verre, Paris, Collection Centre Pompidou, Musée national d'art moderne Centre de création industrielle.

dans les réseaux communistes parisiens et belges, particulièrement autour de Joris Ivens, qui donnent à leur activité cinématographique une vocation éminemment politique (AMAO, 2014, p. 294-301). Les travaux récents de François Albéra et Valérie Vignaux sur Léon Moussinac ont permis également d'établir les liens de proximité entre les photographes du cercle de l'AEAR et le réseau des ciné-clubs (VIGNAUX, 2011 et ALBÉRA, VIGNAUX, 2014). Aussi observe-t-on que c'est dans l'interaction des médias, de la presse et du cinéma, que se forge une culture visuelle spécifiquement liée à l'expression des luttes sociales, d'où l'intérêt d'une approche décloisonnée, attentive aux réseaux de personnes et aux structures associatives ou politiques.

\section{Structures et réseaux transnationaux}

C'est presque une banalité de rappeler que Paris est, à la fin des années 1920, un nœud de réseaux transnationaux. La capitale française n'est d'ailleurs pas, pour beaucoup de ces photographes, l'aboutissement d'un parcours, mais un passage plus ou moins long, une étape sur les sentiers invisibles de la migration transnationale. La photographie sociale partage avec la modernité les origines étrangères que fustigera Emmanuel Sougez (LAVIE, 2013). Germaine Krull, après avoir été engagée très tôt dans la république des conseils à Munich, condamnée en Russie, arrive à Paris en 1928 et poursuit une activité internationale (fig. 8) ; Gisèle Freund débute sa carrière de photographe dans les manifestations du $1^{\text {er }}$ mai 1932 à Francfort-sur-le-Main avant d'émigrer à Paris, où elle poursuit un travail d'historienne en parallèle de son œuvre de portraitiste. Eli Lotar, Roumain de Bucarest arrivé en 1924 à Paris (fig. 9), apprend la photographie auprès de Germaine Krull. Hongrois comme Brassaï, André Kertész arrive de Budapest en 1923, fréquente les surréalistes avant de rejoindre l'AEAR, puis de poursuivre son itinéraire à New York en 1936. Pour certains, le contexte parisien 
n'est d'ailleurs pas celui de la formation photographique. David Seymour, alias Chim, et Robert Capa arrivent avec un fort bagage acquis en Allemagne, l'un à la Hochschule für Graphik de Leipzig, l'autre à la célèbre agence Dephot de Berlin. Il serait vain ici d'allonger la liste. Le catalogue Voici Paris propose une synthèse complète des réseaux étrangers dans le milieu photographique parisien (CHÉROUX, 2012), et montre que seulement $27 \%$ d'entre eux décident de rester dans la capitale après 1940.

Mais, parallèlement aux chemins de l'immigration et de l'exil, les réseaux transnationaux sont tissés par les organisations culturelles internationales. Dans le monde communiste, on vit pour une large part sous l'influence de Moscou (CEURÉ, MAZUY, 2011 ; FAYET, 2014). Plusieurs historiens ont déjà eu l'occasion d'évoquer, à l'instar de Françoise Denoyelle (Denoyelle, 1997, Denoyelle, Cuel, Vibert-Guigne, 2006 ; Denoyelle, 2012a), le rôle de l'AEAR dans les débats autour de la photographie documentaire et sociale dans les années 1930. L'Association des écrivains révolutionnaires (AER) fut créée à la fin de l'année 1931 pour répondre aux critiques émises par les intellectuels soviétiques du congrès de Karkhov (1930) contre Barbusse et la revue Monde - on lui reprochait quelque réticence à défendre la littérature prolétarienne. Ainsi fut mise sur pied la section française de l'Union internationale des écrivains révolutionnaires (UIER) qui deviendra, en y associant les artistes plasticiens en décembre 1932, l'Association des écrivains et artistes révolutionnaires (AEAR). Au sein de cette association se retrouvent des écrivains et des artistes, des architectes, des cinéastes et des photographes, tous proches des idées communistes (BERNARD, 1967). La section photographique mise en place par Aragon et Eli Lotar, secrétaire de la section, comprenait notamment Henri Cartier-Bresson, Henri Tracol, Brassaï, Dora Maar, André Papillon, Jacques-André Boiffard, Pierre Jamet, Claude Cahun, André Kertész, Pierre Ichac, Jean Painlevé, René Zuber.

Les travaux menés depuis les années 1980 permettent de se faire une bonne idée de la question des rapports entre l'art et la politique. Nicole Racine et Serge Fauchereau ont étudié notamment " la querelle du réalisme ", montrant les débats homériques menés en mai 1936 dans l'effervescence de l'élection du Front populaire (FAUCHEREAU, 1987 ; LAHANQUE, 2002 ; RACINE, 2003). Ils ont analysé les différentes positions des artistes peintres, entre " réalisme critique ", "réalisme socialiste " ou " nouveau réalisme " - l'expression est de Fernand Léger. Ils ont étudié le rôle joué par Aragon comme médiateur de positions antagonistes au sein de l'AEAR. Mais il reste que la photographie, en raison même de son statut marginal, n'a pas réellement été intégrée à ces questionnements des historiens. Il ressort pourtant des études récentes que son rôle n'y fut pas secondaire et qu'elle apparut au contraire centrale quand il s'est agi de trouver une réplique à l'échec relatif du Salon des artistes prolétariens de 1934. L'année suivante est organisée l'exposition " Documents de la vie sociale " à la galerie de la Pléiade, saluée et commentée par Aragon dans Commune ${ }^{2}$ (Voici Paris..., 2012-2013; VerKest, 2015).

Là où l'histoire s'est souvent contentée de monographies idéalisant l'engagement individuel des photographes, l'histoire de l'AEAR apparaît comme un contrepoint intéressant, montrant combien ce mouvement collectif fut puissant dans son rôle de catalyseur des débats et des pratiques photographiques. On voit bien comment la recherche d'un réalisme s'accentue pour finalement se dégager du fond surréaliste dont était pourtant issue une bonne partie des membres photographes de l'AEAR. Détournée du vocabulaire de Bataille pour rejoindre celui d'Aragon dans sa défense du réalisme, la notion de document peut être perçue dans ce contexte comme un butin de guerre. Dans ce contexte où la photographie est mise en avant comme art des masses, John Heartfield, figure incontournable du photomontage politique, est introduit en France (KRIEBEL, 2014) avec une rétrospective à la Maison de la culture en 1935. Il apparaît aux yeux d'Aragon comme l'artiste des masses par excellence, celui qui fait 
de la révolution sa poésie, celui dont l'efficace politique se mesure à la violence des réactions de ses ennemis ${ }^{3}$. Il reste encore beaucoup à étudier de ce réseau de photographes engagés dans la gauche radicale, au croisement de l'histoire de la photographie, de la presse et du cinéma. Quel rôle la section photographique de l'AEAR a-t-elle joué pour la consolidation d'une tradition documentaire et sociale ? Quels étaient précisément les liens entre photographie et cinéma au sein de l'AEAR ? Jusqu'à quel degré peut-on parler d'influence allemande ou soviétique sur les structures et les pratiques de ces photographes?

\section{Les « autres " photographes}

Si la photographie paraissait, au sein de l'AEAR et dans les cercles de la gauche et de l'extrême gauche, comme un médium propice à la formation d'une culture visuelle des masses, c'est aussi parce qu'elle incarnait la possibilité d'une appropriation par les classes laborieuses des outils de production visuels, en somme d'un renversement de ce qui était perçu comme la domination symbolique de la " bourgeoisie " sur le " prolétariat ». Bien avant d'être portée au sein de l'AEAR, notamment par Henri Tracol, dans un texte court intitulé "La photographie, une arme de classe ${ }^{4}$ ", l'argument d'une photographie prolétarienne fut développé dans les Associations de photographes ouvriers ou Amateurs photographes ouvriers (les APO ; The Worker Photography Movement..., 2011). Les cercles d'amateurs ou correspondants ouvriers ne partageaient toutefois pas les mêmes orientations que les photographes de l'AEAR, portés vers la reconnaissance de la photographie d'auteur plutôt que vers l'anonymat revendiqué par les APO (ALLAIN, 2015). Organisations militantes de correspondants ouvriers, indépendantes des cercles intellectuels, les APO n'avaient que peu d'affinités avec l'élitisme de l'AEAR. Pour en comprendre la constitution, les objectifs et les réalisations, il faut replacer l'histoire des APO sur le plan international.

Il est indéniable qu'on trouve en Allemagne le noyau dur du mouvement ${ }^{5}$. Créé à l'initiative de Willi Münzenberg, député communiste au Reichstag, cadre du Komintern et chef du Secours ouvrier international, ce mouvement dépendait en partie des organisations communistes. Münzenberg avait débuté ses activités d'agitation avant la guerre en animant l'organisation de jeunesse Freie Jugend et avait été repéré par Lénine lors de son exil à Zurich en 1916 (GROSS, 1967 ; MCMEEKIN, 2003 ; DUGRAND, LAURENT, 2008). Après la guerre, il rejoignit le parti communiste allemand peu de temps après sa création, tout en poursuivant sa voie propre, marquée par un intérêt pour les intellectuels et la propagande. Le Secours ouvrier international, créé en 1921 en principe pour venir en aide aux victimes de la famine soviétique, devint rapidement une entreprise puissante d'agit-prop, soutenue davantage par le Komintern que par le parti allemand. C'est au sein de cette organisation que furent organisés les grands événements sportifs et culturels, que furent édités certains titres de la presse illustrée communiste, notamment l'Arbeiter Illustrierte Zeitung (WILLMANN, 1974), et finalement produits et diffusés quelques grands films soviétiques : Poudovkine, Eisenstein et d'autres eurent partie liée avec les maisons de production de Münzenberg, Mejrabpom Rus et Prometheus Film (AGDE, SCHWARZ, 2012).

Dans cette nébuleuse d'organisations, les photographes ouvriers représentaient ce qu'on pourrait appeler un opérateur de transformation fonctionnelle entre un " capital militant ${ }^{6}$ ", accumulé à l'époque de son engagement dans les mouvements de jeunesse, et une infrastructure médiatique créée plus tard (HESSE, 2009 et 2013, Das Auge des Arbeiters..., 2014). Riche de son expérience de la mobilisation, Münzenberg avait pu observer l'utilité des loisirs et du sport pour l'agit-prop. Avec les photographes ouvriers, il découvrait une autre fonction. En plus de resserrer les liens entre les structures partisanes et le monde associatif, 
les photographes ouvriers se voyaient intégrés au sein d'un système de propagande et d'édition : avec Mejrabpom Rus, sa maison de production de films, et le Neuer Deutscher Verlag, sa maison d'édition, Münzenberg contrôlait un consortium de production et de diffusion de médias auquel il ne manquait qu'une agence photographique. Aussi décida-t-il de s'appuyer sur des bénévoles (JOSCHKE, 2017a et b). À l'avantage économique s'ajoutait celui de fédérer ainsi un réseau de fidèles lecteurs et d'agitateurs, ce qui fut la tâche des rédacteurs de l'AIZ, en particulier celui de Franz Höllering puis, après 1928, de Babette Gross et Lilly Corpus, enfin, de Münzenberg lui-même et des cadres de la fédération des associations allemandes de photographie ouvrière (Verband deutscher Arbeiter-Fotografen-Vereine), notamment Willi Zimmermann, Erich Rinka et Eugen Heilig.

\section{Le rôle de Moscou}

Si l'historiographie n'a jamais fait mystère du soutien du Komintern au mouvement des photographes ouvriers, notre connaissance des rapports entre les photographes prolétariens soviétiques, allemands et français est longtemps restée vague et, de ce fait, leurs connexions exagérées par l'effet d'une presse ouvrière prompte à mettre en avant la solidarité entre les mouvements ouvriers européens et l'URSS. Il existait en URSS un mouvement semblable de photographes amateurs ouvriers, fédérés par la revue Sovietskoe Foto - intitulée Proletarskoe Foto entre 1931 et 1934, avant de revenir à son titre initial (MELOT-HENRY, 2012). Apparu en 1926, en même temps que la fédération allemande des photographes ouvriers, le mouvement de photographie ouvrière d'Union soviétique (ODSK) avait pour but officiel de s'émanciper des cercles bourgeois de photographie pictorialiste. Le magazine Sovietskoe Foto, destiné exclusivement aux amateurs jusqu'en 1931 - il s'adressera alors aussi aux professionnels -, les encourageait, prodiguait des conseils techniques et louait le rôle des correspondants photo-

10. Der Arbeiter-
Fotograf,
$2^{\mathrm{e}}$ année, $\mathrm{n}^{\circ} 7$,
décembre 1927,
première
de couverture,
Dresde,
Sächsische
Landesbibliothek
- Staats- und
Universitäts-
bibliothek
Dresden.

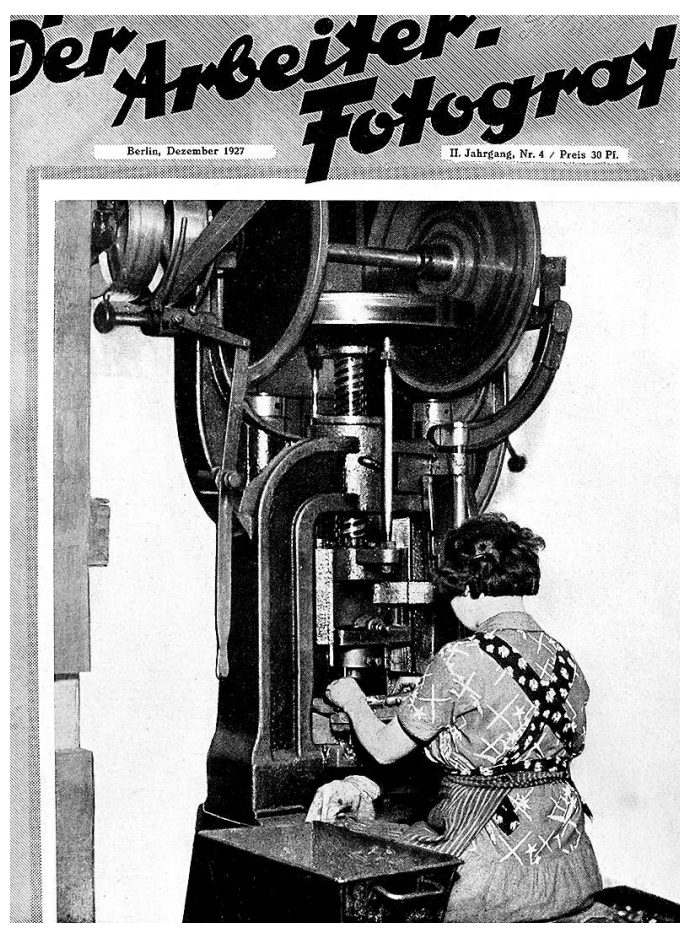
graphes pour la presse illustrée. Cependant, comme l'a bien montré Emily Evans, il ne s'agissait pas d'un mouvement de masse (EVANS, 2014 ; ZAKOVYRINA, 2008). Il n'existait aucun appareil produit en URSS avant la sortie du "FED » en 1933, une imitation russe du Leica à laquelle on avait donné le doux nom de Feliks Edmundovič Dzeržinskij, feu le chef du Guépéou (mort en 1926). Aussi le matériel restait-il inaccessible pour un club d'ouvriers. Et si Grigorij Boltjanskij, le principal animateur de ces associations, avait l'ambition de fédérer un mouvement à l'échelle internationale, les clubs et leurs membres demeuraient peu nombreux.

Néanmoins, les journaux allemands font invariablement référence à la l'Union Soviétique. Les récentes recherches menées par Ursula Schlude ont établi que les liens entre Berlin et Moscou avaient été réels dans ce domaine, bien que surestimés (SCHLUDE, 2012 et 2013). L'ODSK a été créée à Moscou en 1926 par Grigorij Boltjanskij en présence 
de l'Allemand Erich Rinka. En 1927, elle tenta sans succès de fédérer une union internationale impliquant des Français, des Suédois, des Néerlandais, en plus des Russes et des Allemands et, en 1931, une semblable réunion de cadres venus de tous les pays fut convoquée à Moscou, avec aussi peu de résultats ${ }^{7}$. Cependant, il faut constater des échanges réguliers entre l'Allemagne et l'Union Soviétique. Le bulletin Der Arbeiter Fotograf publiait régulièrement en une des photographies envoyées d'URSS (fig. 10), et certains articles de Boltjanskij furent traduits en allemand. On trouve également des références aux reportages ouvriers soviétiques dans le magazine $A I Z$, notamment avec le reportage très médiatisé sur les Filipov, une famille ouvrière " typique " de Moscou, cité par les amateurs comme le modèle d'un genre nouveau de photoreportage, l' " essai photographique " (foto-otcherk). Néanmoins, comme l'a bien montré Erika Maria Wolf, loin s'en fallut que ce reportage ne fût une production soviétique (WOLF, 2011). Cette pure construction de propagande, à l'heure de la collectivisation forcée et de l'exode rural, fut commandée par le Secours ouvrier international de Münzenberg à l'agence soviétique Soyouzfoto pour une exposition montrée à Vienne, à Prague et à Berlin. La série fut préparée par les photographes soviétiques et les cadres de l'AIZ, sans doute John Heartfield lui-même, publiée d'abord en allemand en septembre 1931 dans l'AIZ puis reprise dans le journal Proletarskoe Foto, commentée dans Pravda et enfin dans Regards.

Le modèle soviétique de la photographie ouvrière est donc en grande partie un mythe construit par les Allemands pour resserrer les liens entre Berlin et Moscou. Mais le projet soviétique d'une organisation internationale de photographie ouvrière prit fin avec la mise au pas de la société civile par Staline (SCHLUDE, 2013). L'ODSK fut liquidée en 1932, la revue Sovietskoe Foto allait continuer son activité comme revue de professionnels et d'amateurs éclairés abandonnant la référence à l'agit-prop, et Boltjanskij poursuivit une carrière de critique de cinéma au service de Staline. Le mouvement en Allemagne s'en trouva considérablement affaibli, avant même le coup de grâce infligé par Hitler.

\section{La spécificité française}

La situation en France paraît dans ce contexte bien mal étudiée. Il est vrai que la culture de l'agit-prop ne s'y est pas développée comme en Allemagne. En l'absence de sources manuscrites ou publiées sur les Amateurs photographes ouvriers (APO), sans véritable fonds iconographique, les historiens en sont venus à penser que ces clubs de photographes, pourtant cités dans Der Arbeiter-Fotograf en 1931 et dans le magazine Regards dès 1932, n'avaient jamais vraiment existé. La France ferait figure d'exception à côté de la Hongrie, de la Tchécoslovaquie, de l'Autriche, de l'Espagne, du Portugal ou de l'Angleterre, étudiés par Jorge Ribalta, qui avaient chacun leur mouvement de photographie ouvrière (The Worker Photography Movement..., 2011). Simon Dell explique cette exception française en évoquant la voie particulière du communisme français, détourné, dans sa troisième phase, de la révolution, et porté vers l'union antifasciste (DELL, 2011). Le mouvement des photographes ouvriers en Allemagne serait lié à une lutte des classes qui, dans les milieux culturels français, ne s'est pas manifestée de manière aussi radicale. Mais il ne faudrait pas sous-estimer l'existence de groupes d'ouvriers-photographes dans l'entourage des organes proches du PCF, de l'Almanach ouvrier et paysan, du magazine illustré Regards et de l'Association des écrivains et artistes révolutionnaires (AEAR).

Les APO furent encouragés par le cercle de Léon Moussinac, critique de cinéma à L'Humanité, créateur des ciné-clubs au début des années 1920, ami très proche de Paul VaillantCouturier et directeur de Regards. Les recherches de Valérie Vignaux ont montré son action dans le domaine du cinéma et, par incidence, de la photographie (VIGNAUX, 2011 et 2014). En 1930, Moussinac décida de modifier la structure des ciné-clubs, jusque-là relativement 
élitaires, en les ouvrant au public populaire : c'étaient désormais les Amis de Spartacus. Le critique de L'Humanité ouvrit dans le quotidien une rubrique publiant les avis des spectateurs, à l'image des "boîtes aux lettres " de lecteurs. Parallèlement, furent créées les APO, l'organe associatif des photographes amateurs ouvriers qui, en 1931, se rapprochèrent de la Fédération Ciné-Photo, elle aussi dirigée par Moussinac, et qui avait mené plusieurs expériences de cinéma ouvrier. Et au moment de la création de l'Association des écrivains et artistes révolutionnaires en 1932, Moussinac prit la responsabilité de la section cinéma qui comprenait de facto de nombreux photographes. Les APO déménagèrent alors leur siège du 28 rue Boyer au 8 avenue Mathurin-Moreau puis au 13 rue du Faubourg Montmartre pour se rapprocher de l'AEAR, et devinrent également partie de la Confédération culturelle ouvrière (DELL, 201 1, p. 358). Le nom de Moussinac apparaît d'ailleurs dans les archives Boltjanskij à Moscou, dans le contexte de deux réunions concernant le projet de créer une fédération internationale des photographes ouvriers. À deux reprises, en 1927, puis en 1931, Moussinac absent se vit contraint d'expliquer par lettre que le mouvement manquait d'ampleur dans son pays ${ }^{8}$. Il semble donc que ces associations aient été plus proches des milieux culturels et des ciné-clubs que des mouvements syndicaux ou du loisir sportif, de sorte que l'accent mis par l'AEAR sur la culture plutôt que sur l'agit-prop a pu freiner les velléités prolétariennes des APO. La mise au pas des associations en URSS et le manque de soutien du Komintern à cette époque explique sans doute aussi cette faiblesse du mouvement des photographes ouvriers en France. Son importance culturelle n'en était pourtant pas moins grande, dans la mesure où ses mots d'ordre et le discours qu'il véhiculait sur la photographie comme médium populaire fut décisif pour l'avènement d'une tradition française de la photographie documentaire et sociale.

On voit bien, au terme de ce rapide examen de l'historiographie récente, que pour devenir sociale, puis humaniste, la photographie de l'entre-deux-guerres a d'abord dû être politique, autrement dit, qu'on ne peut comprendre l'émergence de cette tradition qu'en la situant dans

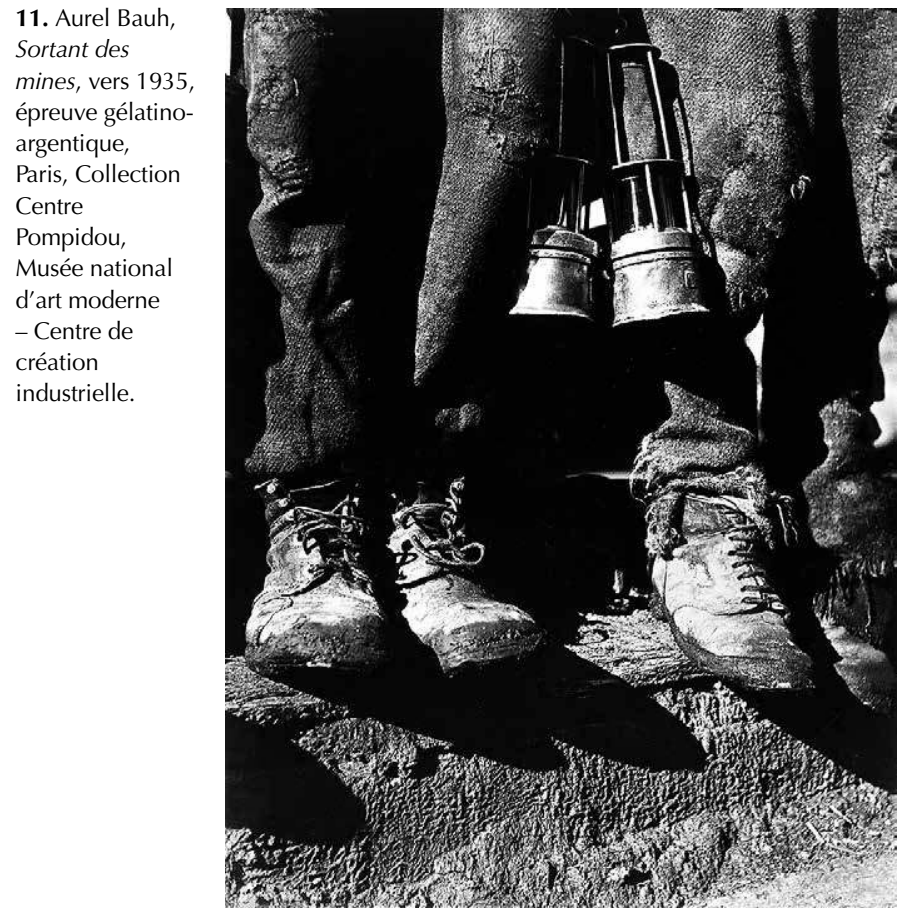
un large contexte qui touche à l'histoire des organisations d'intellectuels, des réseaux de militants, de la presse d'opinion comme de la peinture et du cinéma. La notion de culture visuelle prend ici un sens précis, tangible, tant sur le plan matériel - celui des supports - que des points de vue social et institutionnel - celui des acteurs et des organisations -, elle incarne non seulement un discours mais des pratiques, des dispositifs de capture et de dissémination des images politiques. Les trois directions données par cet élargissement du champ de réflexion, l'intermédialité, l'histoire transnationale et l'ouverture aux "autres photographes" témoignent bien de l'évolution des enjeux : de l'histoire de la modernité photographique, focalisée sur la question de sa reconnaissance comme moyen d'expression artistique et sur la mise en ouvre d'un langage formel abstrait, on est passé à la question des moyens techniques et culturels engagés dans la construction collective d'une 
conscience sociale, à la fois reflet des rapports sociaux et moteur de leur transformation. Ce n'est pas ici un affaiblissement d'une démarche formaliste en histoire de l'art, mais bien une redéfinition du statut de la forme dans un ensemble plus large qui comprend la fonction, le statut, les pratiques et les matières. C'est d'ailleurs peut-être là que se loge une autre modernité, celle qui considère le passage à l'âge contemporain comme la fin de l'intentionnalisme esthétique. L'histoire de la photographie de l'entre-deux-guerres n'est plus seulement l'étude de consciences imageantes, d'individualités créatrices, mais l'analyse des structures, des réseaux et des techniques où les individus producteurs font émerger un regard nouveau sur les transformations du monde.

Ce texte s'appuie sur une recherche menée à l'université Paris Nanterre en collaboration avec le Centre Pompidou, dans le cadre du LabEx Arts H2H. Je tiens à remercier vivement Clément Chéroux, Karolina Lewandowska, Damarice Amao et Julie Jones, ainsi que nos collaboratrices et collaborateurs, Max Bonhomme, Mathilde Esnault, Gabrielle de la Selle et Eva Verkest.

\section{Notes}

1. Carlo Rim, Le grenier d'Arlequin : journal 1916-1940, Paris, 1981.

2. Louis Aragon, "Un "Salon" photographique ", dans Commune, vol. 22, 1935, p. 1189-1192.

3. Louis Aragon, "John Heartfield et la beauté révolutionnaire", dans Commune, vol. 21, 1935, p. 985-991.

4. Henri Tracol, " La photographie, arme de classe ", dans Cahier rouge, vol. 1933, n. p.

5. Il est difficile de se faire une idée de l'ampleur du mouvement de clubs de photographes ouvriers communistes affiliés au Verein der Arbeiter Fotografen Deutschlands. Glienicke, correspondant de Stuttgart présent à Moscou lors d'une réunion en 1931, parle de 35 membres dans l'association de Stuttgart et 400 photographes ouvriers en Allemagne.

6. Frédérique Matonti, Frank Poupeau, " Le capital militant. Essai de définition ", dans Actes de la recherche en sciences sociales, vol. 155, n 5, 2004, p. 4-11.

7. Moscou, RGALI, Fonds Boltjanskij 2057, Opis 1, No 15, p. 15-30, sténogramme de la réunion du bureau international des photo-amateurs le 15 novembre 1927 et Opis 1, n ${ }^{\circ} 13$, p. 155-158, sténogramme de la réunion des photo-correspondants du Caucase du Nord le 16 décembre 1931.

\section{Ibidem.}

\section{Bibliographie}

- Agde, Schwarz, 2012 : Günter Agde, Alexander Schwarz (dir.), Die rote Traumfabrik: Meschrabpom-Film und Prometheus (1921-1936), David Drevs (trad. all.), Berlin, 2012.

- Albera, VignauX, 2014 : François Albera, Valérie Vignaux (dir.), Léon Moussinac : un intellectuel communiste, critique et théoricien des arts, Paris, 2014.

- Allain, 2015 : Patrice Allain, « Du poétique au politique : les épreuves de la révolution ", dans Claude Cahun et ses doubles, cat. exp. (Nantes, médiathèque JacquesDemy, 2015), Nantes, 2015, p. 86-94.

- AMAO, 2014 : Damarice Amao, Passion et désillusion. Eli Lotar (1905-1969). Contribution à une histoire des rapports entre les avantgardes photographique et cinématographique à Paris dans l'entre-deux-guerres, thèse de doctorat, université Paris Sorbonne, 2014. - Das Auge des Arbeiters..., 2014 : Das Auge des Arbeiters. Arbeiterfotografie und Kunst um 1930 , Wolfgang Hesse (dir.), cat. exp. (Zwickau, Kunstsammlungen, Max-Pechstein-Museum/Cologne, Käthe Kollwitz Museum/ Dresde, Stadtmuseum Dresden, Museen der Stadt Dresden, 2014-2015), Leipzig, 2014. - Avantgarde als..., 1999 : Avantgarde als Abenteuer: Leben und Werk der Photographin Germaine Krull, Kim Sichel (dir.), cat. exp. (Essen Museum Folkwang/
Paris, Centre Pompidou - Musée national d'art moderne, 1999), Munich, Schirmer/ Mosel, 1999.

- BAJAC, 2012 : Quentin Bajac, « Paris à la découverte de la photographie moderne, 1929-1939 ", dans Voici Paris..., 20122013, p. 16-29.

- BENJAMIN, 1991 : Walter Benjamin, Écrits français, Jean-Maurice Monnoyer (éd.), Paris, 1991.

- BERNARD, 1967 : Jean-Pierre A. Bernard, "Le Parti communiste français et les problèmes littéraires (1920-1939)", dans Revue française de science politique, 1967, p. 520-544.

- BERNARD, 1991 : Jean-Pierre A. Bernard, Paris rouge. 1944-1964. Les communistes français dans la capitale, Paris, 1991.

- Bool, 1982 : Flip Bool, De arbeidersfotografen: camera en crisis in de jaren ' 30 , Amsterdam, 1982.

- Brassaï, graffiti..., 2016 : Brassaï, graffiti. Le langage du mur, Karolina ZiebinskaLewandowska (dir.), cat. exp. (Paris, Centre Pompidou - Musée national d'art moderne, Galerie de photographies, 20162017), Paris, 2016.

- CARR, 1983 : Edward Hallet Carr, The Twilight of the Komintern, 1930-1935, Londres, 1983.

- Henri Cartier-Bresson, 2013 : Henri CartierBresson, Clément Chéroux (dir.), cat. exp. (Paris, Centre Pompidou - Musée national d'art moderne/Madrid, Instituto de Cultura-Fundacion Mapfre, 2014), Paris, 2013. - Challine, 2014 : Éléonore Challine, "La critique photographique des années trente en France ", dans Revue Française d'Histoire des Idées Politiques, vol. 39, $\mathrm{n}^{\circ} 1$, 2014, p. 27-39. 
- CHÉroux, 2012 : Clément Chéroux, «Du cosmopolitisme en photographie. Portrait de Paris en échangeur culturel ", dans Voici Paris..., 2012, p. 30-41.

- Chominot, 2001 : Marie Chominot, Les Photographies du Front populaire épisode humaniste -à travers la presse illustrée et quotidienne (1936-1937). La construction d'un imaginaire photographique de l'histoire, mémoire de maîtrise, Paris 1 PanthéonSorbonne, 2001.

- Coevré, Mazuy, 2011 : Sophie Couré, Rachel Mazuy (dir.), Cousu de fil rouge: voyages des intellectuels français en Union soviétique. 150 documents inédits des archives russes, Paris, 2011.

- Courtois, 1994 : Stéphane Courtois, " La seconde mort de Willy Münzenberg ", dans Communisme. Revue du Centre d'étude d'histoire et de sociologie du communisme, $\mathrm{n}^{\circ}$ 38-39, dossier spécial « Les Kominterniens ", 1994, p. 25-44.

- Cuevas-Wolf, 2008 : Cristina CuevasWolf, " A Defiant Spain: Münzenberg, Montage and Communist Media in France and Spain ", dans Mendelson, 2008, p. 55-73.

- Cuevas-Wolf, 2009 : Cristina CuevasWolf, « Montage as Weapon: The Tactical Alliance between Willi Münzenberg and John Heartfield ", dans New German Critique, $\mathrm{n}^{\circ} 107,2009$, p. 185-205.

- D’Almeida, Delporte, 2003 : Fabrice d'Almeida, Christian Delporte, L'histoire des médias en France : de la Grande Guerre à nos jours, Paris, 2003.

- DeLL, 2000 : Simon Dell, "Festival and Revolution: the Popular Front in France and the Press Coverage of Strikes of 1936 ", dans Art History, vol. 23, n 4 , novembre 2000, p. 599-621.

- DeLl, 2001 : Simon Dell, « On the Metaphor and Practice of Photography: Socialist Realism, the Popular Front in France and the Dynamics of Cultural Unity ", dans History of Photography, vol. 25, $\mathrm{n}^{\circ} 1$, printemps 2001, p. 52-60.

- DELL, 2003 : Simon Dell, «Internationalismus und die Kultur der Volksfront: John Heartfield und die Fotomontage ", dans Volker Zaib(dir.), Kultur als Fenster zu einem besseren Leben und Arbeiten: Festschrift für Rainer Noltenius, Bielefeld, 2003, p. 369-384.

- DeLL, 2011 : Simon Dell, "The Difficult Conjunction Between "Worker" and "Photographer" in France ", dans The Worker Photography Movement..., 2011, p. 354-362. - Denoyelle, 1997 : Françoise Denoyelle, Lumière de Paris, 1. Le marché de la photographie : $1919-1939 ; 2$. Les usages de la photographie : 1919-1939, Paris, 1997, 2. vol.
- DeNOyelle, 2012a : Françoise Denoyelle, "Amitiés et réseaux des photographes parisiens dans les années 1930 ", dans Voici Paris..., 2012-2013, p. 42-55.

- Denoyelle, 2012b : Françoise Denoyelle, Le Siècle de Willy Ronis, Paris, 2012.

- Denoyelle, Cuel, Vibert-Guigne, 2006 : Françoise Denoyelle, François Cuel, Jean-Louis Vibert-Guigue, Le Front populaire des photographes, Paris, 2006.

- DicKeRMAN, 2006 : Leah Dickerman,

"The Fact and the Photograph ", dans October, vol. 118, 2006, p. 132-152.

- Didi-Huberman, 2009 : Georges DidiHuberman, L'ail de l'histoire, I. Quand les images prennent position, Paris, 2009.

- Dugrand, Laurent, 2008 : Alain Dugrand, Frédéric Laurent, Willi Münzenberg : artiste en révolution, 1889-1940, Paris, 2008.

- EGGERT, 1989 : Christine Eggert, Zu Geschichte und Wirken der Assoziation Revolutionärer Bildender Künstler Deutschlands/ Bund Revolutionärer Bildender Künstler Deutschlands und Berücksichtigung von Erinnerungen ehemaliger Mitglieder Berliner Ortsgruppe, thèse de doctorat, Université de Berlin, 1989.

- Encounters with the 30s, 2012-2013: Encounters with the 30s, Jordana Mendelson (dir.), cat. exp. (Madrid, Museo nacional Centro de arte Reina Sofía, 2012-2013), Madrid, 2012.

- ESKILDSEN, 1980 : Ute Eskildsen, «The A-I-Z and the Arbeiter Fotograf: Working Class Photographers in Weimar ", dans Image, vol. 23, $\mathrm{n}^{\circ} 2,1980$, p. 1-8.

- EsNAult, 2015 : Mathilde Esnault, La Photographie documentaire et sociale au sein de la revue Regards. 1928-1939, thèse de doctorat, université Paris Nanterre, 2015. - Evans, 2014 : Emily Joyce Evans, Soviet Photo and the Search for Proletarian Photography, 1926-1937, thèse de doctorat, University of Illinois at Urbana-Champaign, 2014. - EvANs, 2015 : Emily Joyce Evans, " Hinwendung und Abkehr vom Arbeiterporträt. Brüche und Kontinuitäten in der Entwicklung des "sowjetischen" Porträts 1926-1937 ", dans Fotogeschichte, n 136, 2015, p. 33-42.

- FAUChereau, 1987 : Serge Fauchereau et al., La Querelle du réalisme, Paris, 1987.

- FAYET, 2014 : Jean-François Fayet, VOKS : le laboratoire helvétique. Histoire de la diplomatie culturelle soviétique durant l'entredeux-guerres, Chêne-Bourg, 2014.

- FORE, 2006 : Devin Fore, "The Operative Word in Soviet Factography ", dans October, vol. 118, 2006, p. 95-131.
- FORE, 2012 : Devin Fore, Realism after Modernism. The Rehumanization of Art and Literature, An October Book, Cambridge, Mass., 2012.

- Fotografía pública..., 1999-2000 : Fotografía pública / Photography in Print, 19191939, Horacio Fernandez (dir.), cat. exp. (Madrid, Museo Nacional Centro de Arte Reina Sofia/Bilbao, Museo de Bellas Artes/ Logronõ, Centro Cultural La Rioja, 19992000), Madrid, 1999.

- Gisele Freund..., 1995 : Gisele Freund. Fotografien, cat. exp. (Francfort-sur-le-Main, Museum für Moderne Kunst, 1995), Francfort-sur-le-Main, 1995.

- FREUnD, 2011 : Gisèle Freund, Carnets de Gisèle Freund, éd. fac-similé, Paris, 2011.

- FrIzOT, 2010 : Michel Frizot, "D’un imaginaire cinéma de la photographie (1928-1930) ", dans GUIDO, LUGON, 2010 , p. 203-224.

- Frizot, 2014 : Michel Frizot, "The Poetics of Eye and Lens ", dans Mitra Abbaspour et al. (dir.), Object: Photo. Modern Photographs: The Thomas Walther Collection 1909-1949. An Online Project of The Museum of Modern Art, New York, 2014, p. 1-25. - FRIZOT, 2015 : Michel Frizot, "Sports photomontage, France 1926 ", dans Jason Hill, Vanessa Schwartz (dir.), Getting the Picture: the Visual Culture of the News, Londres/ New York, 2015, p. 44-47.

- Frizot, Veigy, 2009 : Michel Frizot, Cédric de Veigy, $V u$ : le magazine photographique, 1928-1940, Paris, 2009.

- GABRIEL, 2012 : Nicole Gabriel, « Le Studio Mejrabpom-Russ : L'usine à rêves des Rouges ? (1935-1936) ", dans Jeune Ciné$m a, \mathrm{n}^{\circ} 344-45$, printemps 2012, p. 94-102. - GERVAIS, 2008 : Thierry Gervais, L'illustration photographique. Naissance du spectacle de l'information, thèse de doctorat, EHESS, 2008.

- GERVAIS, MOREL, 2015 : Thierry Gervais, Gaëlle Morel, La fabrique de l'information visuelle. Photographies et magazines d'actualité, Paris, 2015.

- GOLAN, 2012 : Romy Golan, " La possibilité d'un photomural socialiste ", dans Maria Stavrinaki, Maddalena Carli (dir.), Artistes et partis : esthétique et politique (19001945), Dijon, 2012, p. 231-252.

- Gough, 2002 : Maria Gough, "Paris, Capital of the Soviet Avant-Garde ", dans October, vol. 101, été 2002, p. 53-83.

- GoUgh, 2005 : Maria Gough, The Artist as Producer. Russian Constructivism in Revolution, Berkeley, 2005.

- Gough, 2009 : Maria Gough, "Back in the USSR: John Heartfield, Gustavs Klucis, and the Medium of Soviet Propaganda ", 
dans New German Critique, n ${ }^{\circ} 107,2009$ p. 133-183.

- Gough, 2012 : Maria Gough, "Tretjakovs fotografische Utopie des Faktischen ", dans Wolfgang Hesse (dir.), Die Eroberung der beobachtenden Maschinen, Leipzig, 2012 p. 159-186.

- Gough, Roloff, 2006 : Maria Gough, Michael Roloff, " Radical Tourism: Sergei Tret'iakov at the Communist Lighthouse ", dans October, vol. 118, 2006, p. 159-178. - Gross, 1967 : Babette Gross, Willi Münzenberg. Eine politische Biographie, Stuttgart ( " Schriftenreihe der Vierteljahreshefte für Zeitgeschichte ", nº 14-15), 1967.

- GUIDO, LUGON, 2010 : Laurent Guido, Olivier Lugon, Fixelanimé : croisements de la photographie et du cinéma au $X X^{e}$ siècle, Lausanne, 2010.

- Albert Hennig..., 2007 : Albert Hennig, 1907-1998. Fotografien 1928-1933, Wolfgang Hesse (dir.), cat. exp. (Zwickau, Städtische Museen Zwickau, Kunstsammlungen, 2007), Zwickau, 2007.

- Hesse, 2009 : Wolfgang Hesse, « Der Amateur als politischer Akteur ", dans Fotogeschichte, vol. 111, 2009, p. 21-30.

- Hesse, 2012 : Wolfgang Hesse (dir.), Die Eroberung der beobachtenden Maschinen. Zur Arbeiterfotografie der Weimarer Republik, actes de colloque (Dresde, Staats- und Universitätsbibliothek, 2010), Leipzig, 2012. - Hesse, 2013 : Wolfgang Hesse, Körper und Zeichen. Arbeiterfotografien aus Dohna, Heidenau und Johanngeorgenstadt 1932-33, Dresde, 2013.

- HOFFMANN, 2011 : David L. Hoffmann, Cultivating the Masses. Modern State Practices and Soviet Socialism, 1914-1939, Ithaca/ Londres, 2011.

- Hoffmann-Ostwald, Behse, 1960 : Daniel Hoffmann-Ostwald, Ursula Behse, Agitprop 1924-1933, Leipzig, 1960.

- HogenKamp, 1981 : Bert Hogenkamp, "Le mouvement ouvrier et le cinéma", dans Image et Son, $\mathrm{n}^{\circ} 346$, novembre 1981 , p. 125-135.

- Holzer, 2013 : Anton Holzer, " Vorwärtz! Die österreichische Arbeiterfotografie der Zwischenkriegszeit ", dans Fotogeschichte, $\mathrm{n}^{\circ} 127,2013$, p. 17-30.

- Kati Horna, 2013-2014: Kati Horna, Angeles Alonso Espinosa, José Antonio Rodriguez (dir.), cat. exp. (Puebla, Museo Amparo [Mexique]/Paris, Jeu de Paume, 2013-2014), Paris, 2014.

- Joschke, 2012 : Christian Joschke, " "Bist Du schon Mitglied?" Arbeiterfotografie und Polizeistaat ", dans HESSE, 2012, p. 285-302.
- JoschKe, 2017a : Christian Joschke, "Fotografie als Ware im Klassenkampf. Münzenberg und sowjetische Bildagenturen ", dans Woflgang Hesse, Holger Starke (dir.), Arbeiter | Kultur | Geschichte. Arbeiterfotografie der Weimarer Republik im Museum, actes de colloque (Dresde, Stadtmuseum Dresden, Institut für Sächsische Geschichte und Volkskunde/Hochschule für Technik, Wirtschaft und Kultur Leipzig/Käthe Kollwitz Museum Köln/Kunstsammlungen Zwickau, 2015), Dresde, 2017, p. 115-139. - JoschKe, 2017b : Christian Joschke, " Le marché transnational des images politiques. Le Secours ouvrier international dans le contexte des agences photographiques soviétiques (1924-1933)", dans Études photographiques, n ${ }^{\circ} 35$, mars 2017, p. 3-29.

- Joyeux, 2003 : Marie-Hélène Joyeux, " Le Photomontage politique à travers les couvertures de $V U$ (1928-1936)", dans Les Cahiers du Musée national d'art moderne, $\mathrm{n}^{\circ} 84,2003$, p. 48-65.

- KePLey, 1983 : Vance Kepley Jr., « The Workers' International Relief and the Cinema of the Left 1921-1935", dans Cinema Journal, vol. 23, no 1, 1983, p. 7-23. - KERBS, UKA, 2004 : Diethart Kerbs, Walter Uka (dir.), Fotografie und Bildpublizistik in der Weimarer Republik, Bönen, 2004.

- KolchevsKA, 1987 : Natacha Kolchevska, "From Agitation to Factography: The Plays of Sergej Tret'jakov ", dans The Slavic and East European Journal, vol. 31, no 3, 1987, p. 388-403.

- François Kollar..., 2016 : François Kollar. Un ouvrier du regard, Matthieu Rivallin, Pia Viewing (dir.), cat. exp. (Paris, Jeu de Paume/Bratislava, Slovak National Gallery, 2016), Paris, 2016.

- KRIEBEL, 2014 : Sabine Kriebel, Revolutionary Beauty. The Radical Photomontages of John Heartfield, Berkeley, Ca., 2014.

- Lore Krüger..., 2015 : Lore Krüger: ein Koffer voller Fotos, 1930-1945, Cornelia Bästlein (dir.), cat. exp. (Berlin, C/O Berlin Foundation, 2015), Berlin, 2015.

- Germaine Krull, 2015-2016 : Germaine Krull, Michel Frizot (dir.), cat. exp. (Paris, Jeu de Paume/Berlin, Martin-GropiusBau, 2015-2016), Paris, 2015.

- LAHANQUe, 2002 : Reynald Lahanque, Le Réalisme socialiste en France (1934-1954), thèse de doctorat, université de Nancy, 2002.

- LAVIE, 2013 : Juliette Lavie, Emmanuel Sougez (1889-1972). Un photographe en prise avec son temps, thèse de doctorat, université Paris Nanterre, 2013.
- LeEnEARTs, 2010 : Danielle Leenearts, Petite histoire du magazine VU. Entre photographie d'information et photographie d'art (1928-1940), Bruxelles, 2010.

- LORZ, 2012 : Korinna Lorz, " Das Dorf im Blick. Arbeiterfotografie im Westerzgebirge ", dans HeSSE, 2012, p. 67-112. - LoRZ, 2013 : Korinna Lorz, " "FotoBauhäuseler, werdet Arbeiter-Fotografen!" Fotografie am Bauhaus zwischen Avantgarde und Agitation. Ein Werkstattbericht ", dans Fotogeschichte, vol. 127, 2013, p. 31-44.

- LoRZ, 2014 : Korinna Lorz, "Albert Hennig, ein Bauarbeiter am Bauhaus ", dans Das Auge des Arbeiters..., 2014, p. 83-96. - Eli Lotar, 2017 : Eli Lotar, Damarice Amao, Clément Chéroux, Pia Viewing (dir.), cat. exp. (Paris, Jeu de Paume, 2017), Paris, 2017.

- LuGON, 1997 : Olivier Lugon (dir.), La photographie en Allemagne. Anthologie de textes (1919-1939), Nîmes, 1997.

- Lugon, 2001 : Olivier Lugon, Le style documentaire. D'August Sander à Walker Evans. 1920-1945, Paris, 2001.

- LUGON, 2009 : Olivier Lugon, « Die globalisierte Ausstellung: The Family of Man, 1955 ", dans Fotogeschichte, vol. 29, n 112 , 2009, p. 65-72.

- LUGon, 2012 : Olivier Lugon, "Das kollektive Auge : die Inszenierung des Kollektivs in der Zeitschrift "Der ArbeiterFotograf" ", dans HESSE, 2012, p. 257-284.

- McMeekin, 2001 : Sean McMeekin, "Le secours ouvrier international : histoire d'une légende ", dans Communisme, vol. 65-66, 2001, p. 117-133.

- MCMEEKIN, 2003 : Sean McMeekin, The Red Millionaire. A Political Biography of Willi Münzenberg, Moscow's Secret Propaganda Tsar in the West, New Haven/Londres, Yale University Press, 2003.

- Melot-Henry, 2012 : Annette MelotHenry, La photographie soviétique de 1917 à 1945. Sources et travaux de la BDIC, Nanterre, 2012.

- Mendelson, 2008 : Jordana Mendelson (dir.), Magazines, Modernity and War, Madrid, 2008.

- MoldeRINGs, 1980 : Herbert Molderings, "René Zuber (1902-1979) ", dans Les Cahiers du Musée national d'art moderne, vol. 3, 1980, p. 70-85.

- MOLDERINGS, 1982 : Herbert Molderings, "Tina Modotti photographe ", dans Les Cahiers du Musée national d'art moderne, vol. 10, 1982, p. 247-259.

- MOLDERINGS, 1995 : Herbert Molderings, Umbo. Otto Umbehr, 1902-1980, Düsseldorf, 1995. 
- MOLDERINGS, 2009 : Herbert Molderings, L'évidence du possible. Photographie moderne et surréalisme, Paris, 2009.

- MOREL, 2001 : Gaëlle Morel, « Du peuple au populisme : les couvertures du magazine communiste Regards (1932-1939)", dans Études photographiques, $\mathrm{n}^{\circ}$ 9, mai 2001 , p. 44-63.

- ORY, 1994 : Pascal Ory, La Belle Illusion. Culture et politique sous le signe du Front populaire (1935-1938), Paris, 1994.

- Roger Parry..., 2007 : Roger Parry : photographies, dessins, mises en pages, Mouna Mekouar, Christophe Berthoud, cat. exp. (Paris, Jeu de Paume, 2007), Paris, 2007. - La photographie humaniste..., 2006 : La photographie humaniste, 1945-1968 autour d'Izis, Boubat, Brassaï, Doisneau, Ronis, Laure Beaumont-Maillet, Françoise Denoyelle, Dominique Versavel (dir.), cat. exp. (Paris, Bibliothèque nationale de France, 2006), Paris, 2006.

- POIVERT, 2006 : Michel Poivert, L'image au service de la révolution : photographie, surréalisme, politique, Cherbourg, 2006.

- Pozner, Kherroubi, 1996 : Valérie Pozner, Aïcha Kherroubi (dir.), Le studio Mejrabpom ou l'aventure du cinéma privé au pays des bolcheviks, Paris, 1996.

- Prost, 2006 : Antoine Prost, Autour du Front Populaire. Aspect du mouvement social au $X X^{e}$ siècle, Paris, 2006.

- RACINE, 2003 : Nicole Racine, " “La Querelle du Réalisme" (1935-1936)", dans Sociétés e Représentations, vol. 1, n 15 , 2003, p. 113-131.

- RANCIÈRE, 1981 : Jacques Rancière, La nuit des prolétaires. L'espace du politique, Paris, 1981.

- Alexander Rodtschenko..., 1999-2000 : Alexander Rodtschenko - Das neue Moskau. Photographien aus der Sammlung $L$. und G. Tatunz, Margarita Tupitsyn (dir.), cat. exp. (Hannover, Sprengel Museum/ Seattle, Seattle Art Museum, 1999-2000), Munich/Paris/Londres, 1998.

- Willy Ronis..., 2010 : Willy Ronis, une poétique de l'engagement, Marta Gili, Nathalie Neumann (dir.), cat. exp. (Paris, Monnaie de Paris, 2010), Paris, 2010.

- ROTH, 2006 : Nancy Roth, " Heartfield's collaboration ", dans The Oxford Art Journal, vol. 29, nº 3, 2006, p. 395-418.

- SACHSSE, 2014 : Rolf Sachsse, " Arbeiterfotomoderne. Die Arbeiterfotografie zwischen politischer Bewegung, Amateurfotografie und Neuem Sehen ", dans Das Auge des Arbeiters..., 2014, p. 73-82.

- SCHLUde, 2012 : Ursula Schlude, " "Es wäre uns peinlich, schlechte Fotos zu schicken." Die Austauschbeziehungen zwischen deutschen und sowjetischen Arbeiterfotografen 1926 bis 1933 ", dans HESSE, 2012, p. 113-158.

- SCHLUdE, 2013 : Ursula Schlude, «Fundstellen in Moskau. Zur Überlieferung der kommunistischen Fotoamateurbewegung in russischen Archiven ", dans Fotogeschichte, vol. 127, 2013, p. 5-16.

- SICHEL, 1989 : Kim Sichel, "Les photographes étrangers à Paris ", dans André Kaspi, Antoine Marès (dir.), Le Paris des étrangers depuis un siècle, Paris, 1989, p. 257 267.

- STANGE, 1989 : Maren Stange, Symbols of Ideal Life. Social Documentary Photography in America, 1890-1950, Cambridge/New York, 1989.

- StANGe, 1990 : Maren Stange (dir.), Paul Strand. Essays on his Life and Work, New York, 1990.

- STUMBERGER, 2007 : Rudolf Stumberger (dir.), Klassen-Bilder. Sozialdokumentarische Fotografie 1900-1945, Constance, 2007.

- TARDIVON, 1999 : Jean-Christophe Tardivon, La photographie politique médiatisée : entre actualité et histoire. Étude de cas dans la presse française du début des années 1930. thèse de doctorat, EHESS, 1999.

- TUPITSYN, 1996 : Margarita Tupitsyn, The Soviet Photograph: 1924-1937, New Haven/ Londres, 1996.

- VERKEST, 2015 : Eva Verkest, L'Exposition photographique "Documents de la vie sociale" de l'Association des artistes et écrivains révolutionnaires à la Galerie de la Pléiade à Paris en 1935, mémoire de master, université Paris Nanterre, 2015.

- VeZYROGLOU, 2012 : Dimitri Vezyroglou, "Le Parti communiste et le cinéma ", dans Vingtième Siècle. Revue d'histoire, n 3, 2012 , p. 63-74.

- VignAUX, 2011 : Valérie Vignaux, "Cinéma militant et militantisme culturel dans l'entre-deux-guerres en France ", dans Études photographiques, vol. 27, mai 2011, p. 200-224.

- VIGNAUX, 2014 : Valérie Vignaux, "Léon Moussinac théoricien du cinéma : d'une poétique des arts à une politique de la culture ", dans ALBERA, VignaUX, 2014, p. 111-188.

- Voici Paris..., 2012-2013 : Voici Paris. Modernités photographiques, 1920-1950. La collection Christian Bouqueret, Quentin Bajac, Clément Chéroux (dir.), cat. exp. (Paris, Centre Pompidou, Galerie d'art graphique et Galerie du musée, 2012-2013), Paris, 2012.
- Wilmann, 1974: Heinz Willmann, Geschichte der Arbeiter-Illustrierten Zeitung 1921-1938, Berlin, 1974.

- WOLF, 1999 : Erika Wolf, USSR in Construction. From Avant-Garde to Realist Socialist Practice, thèse de doctorat, University of Michigan, 1999.

- WOLF, 2011 : Erika Wolf, " "As at the Filippovs": The Foreign Origins of the Soviet Narrative Photographic Essay ", dans The Worker Photography Movement..., 2011, p. 124-130.

- Wolikow, 1996 : Serge Wolikow, Le Front populaire en France, Bruxelles, 1996. - The Worker Photography Movement..., 2011 : The Worker Photography Movement (1926-1939): Essays and Documents, Jorge Ribalta (dir.), cat. exp. (Madrid, Museo Centro de Arte Reina Sofia, 2011), Madrid, 2011.

- ZAKOVYRINA, 2008 : Natalja S. Zakovyrina, Osobennosti razvitija sovetskoj fotozhurnalistiki 1920-1930ch godov i zhurnal "Sovetskoe foto », Saint-Petersbourg, 2008. -ZERVIGón, 2010 : Andrés Mario Zervigón, "Persuading with the Unseen? Die Arbeiter-Illustrierte-Zeitung, Photography and German Communism's Iconophobia ", dans Visual Resources, vol. 26, $\mathrm{n}^{\circ}$ 2, 2010 , p. 147-164.

-ZERVIGÓN, 2012 : Andrés Mario Zervigón, John Heartfield and the Agitated Image. Photography, Persuasion, and the Rise of AvantGarde Photomontage, Chicago, 2012. 